\title{
CNV-WebStore: Online CNV Analysis, Storage and Interpretation
}

\author{
Geert Vandeweyer ${ }^{1}$, Edwin Reyniers ${ }^{1,2}$, Wim Wuyts ${ }^{2}$, Liesbeth Rooms ${ }^{1}$, R Frank Kooy ${ }^{1 *}$
}

\begin{abstract}
Background: Microarray technology allows the analysis of genomic aberrations at an ever increasing resolution, making functional interpretation of these vast amounts of data the main bottleneck in routine implementation of high resolution array platforms, and emphasising the need for a centralised and easy to use CNV data management and interpretation system.

Results: We present CNV-WebStore, an online platform to streamline the processing and downstream interpretation of microarray data in a clinical context, tailored towards but not limited to the Illumina BeadArray platform. Provided analysis tools include CNV analsyis, parent of origin and uniparental disomy detection. Interpretation tools include data visualisation, gene prioritisation, automated PubMed searching, linking data to several genome browsers and annotation of CNVs based on several public databases. Finally a module is provided for uniform reporting of results.
\end{abstract}

Conclusion: CNV-WebStore is able to present copy number data in an intuitive way to both lab technicians and clinicians, making it a useful tool in daily clinical practice.

\section{Background}

Up to $12 \%$ of the humane genome is present in a variable number of copies, referred to as copy number variation (CNV) [1-3]. A small subset of specific CNVs is shown to associate with a wide spectrum of diseases [4-9].

Before the development of array-based comparative genomic hybridisation, visual inspection of karyotypes under the microscope limited the detection of chromosomal aberrations to events larger than 5 to $10 \mathrm{Mb}$. A major breakthrough was achieved with the generation of BAC-arrays, initially consisting of about 2000 largeinsert clones spotted on a glass back plate [10]. With the capacity to screen a whole genome at once at a practical resolution of about $1.4 \mathrm{Mb}$, this type of array launched the $\mathrm{CNV}$-analysis field in routine diagnostics. More recently, the resolution of BAC-arrays was improved using over 20000 tiling probes [2]. At present, $\mathrm{BAC}$-arrays are gradually replaced by higher resolution platforms such as oligonucleotide- and SNP-arrays $[11,12]$. The major advantage of SNP-arrays over

\footnotetext{
* Correspondence: frank.kooy@ua.ac.be

'Department of Medical Genetics, University Hospital Antwerp, Antwerp, Belgium

Full list of author information is available at the end of the article
}

oligonucleotide- and BAC-arrays, is that they provide genotype information in addition to intensity ratios. Combining both information layers gives these platforms the potential to detect CNVs at a significantly higher resolution than the first generation platforms $[13,14]$.

This increased resolution, in combination with the wide variety of data analysis methods, causes a shift of focus from data generation to data interpretation. To interpret these calls in a clinical context several aspects typically need to be taken into account [8] and several tools are available to aid in this process [15-17]. While many tools exist for either data analysis or presentation and interpretation, few exist that efficiently combine both [18-23]. Here, we present CNV-WebStore, a webbased platform that couples data analysis with data storage, visualisation, several downstream analysis methods and reporting tools, to serve as a complete data to report pipeline (figure 1).

The presented platform incorporates a majority vote based analysis pipeline specific for Illumina BeadArray data, capable to detect CNVs with a lower limit of 3 consecutive probes, mosaicism and uniparental disomy. Though analysis of data generated on other platforms is not supported, CNV sets from any platform can be 


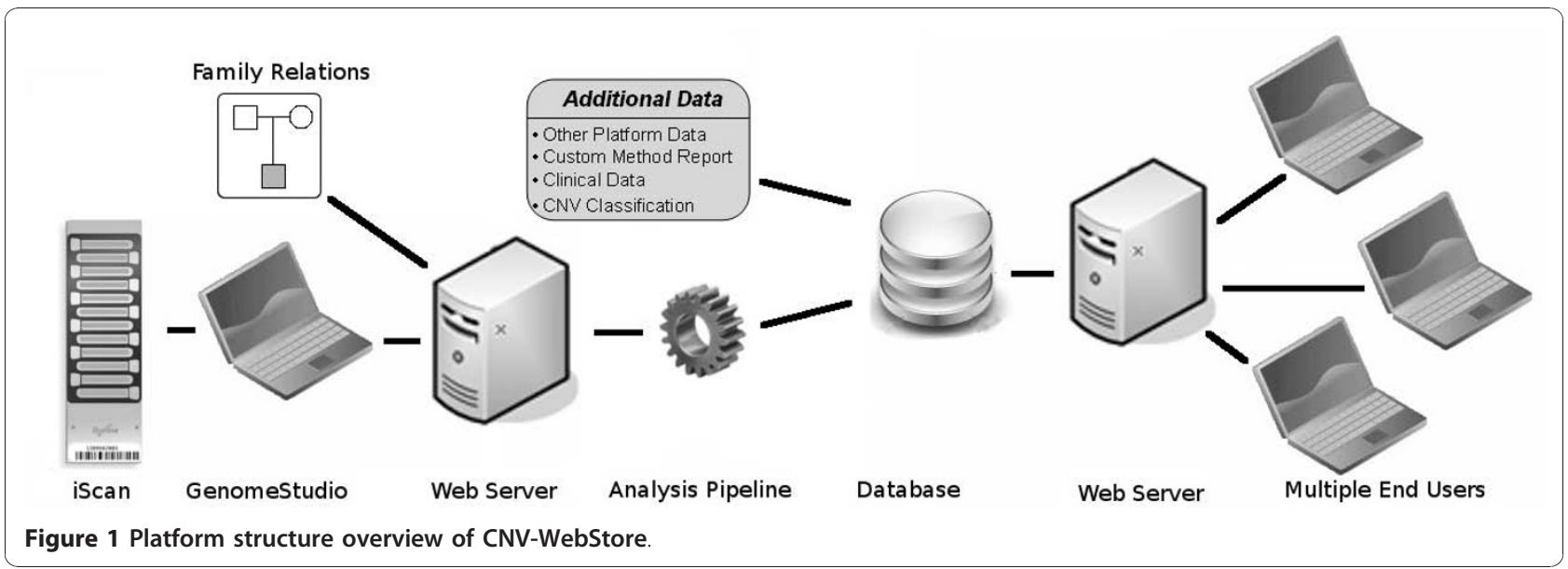

uploaded for downstream analysis. This enables the integration of multiple experimental techniques in a single interpretation pipeline.

\section{Platform Implementation \\ Interface architecture}

CNV-WebStore is hosted on an 8 core, 64bit Suse Linux Enterprise Enterprise Server with 16 Gb RAM, running Apache v2.2.0, PHP v5.2.3 and MySQL v5.1.39. All interface components are composed of PHP and JavaScript, with support for most major browsers. Annotation data is automatically updated on a weekly basis using scheduled Perl scripts. Default data analysis times range from 2 minutes per sample for $300 \mathrm{~K}$ arrays, to 10 minutes for $1 \mathrm{M}$ arrays.

\section{Data analysis packages}

All analysis methods were run in their native programming language in a 64bit Linux environment, and combined by a wrapper script written in Perl v5.8.8 with multithreading support.

QuantiSNP v1.1 was installed and used confirming to the Academic licence. Parameters were set mainly at default values, namely 10 Expectation-Maximisation steps, $2 \mathrm{M}$ as characteristic length for CNVs and a maximal copy number of 4. Additionally, correction for local GC content was applied. Minimal Log Bayes Factor was set at 8.5 based on experimental experience [24].

PennCNV rev081119 was installed and run with the generic markov model and population frequency of B-alleles, since no chip-specific files were available. No family information was used and minimal confidence was set to 10 based on experimental experience [25].

VanillaICE v1.4.0 was installed under $\mathrm{R}$ v2.9.0 and configured according to the authors suggestions for analysing Illumina data. Expected intensity ratios were adapted following technical guidelines from Illumina and emission probability calculations were set to cope with non-polymorphic probes. Samples were analysed individually, so we chose a robust in sample estimate of intensity variability as proposed by the author [26]. A complete overview of the used settings is available as additional file 1.

BAFsegmentation v1.1.0 was installed under R v2.9.0. Program parameters were set to 0.97 as 'informative_treshold', 0.8 as 'triplet_treshold', 0.56 as 'ai_treshold' and 4 as 'ai_size' [27].

SNPtrio [28] calculations were implemented as described by the authors using Perl.

\section{Results}

\section{Data analysis approach}

To make a correct functional interpretation of $\mathrm{CNV}$ data, it is necessary to be able to start from a reliable set of candidate regions. We therefore implemented an analysis pipeline for the Illumina BeadArray platform, aimed at detecting rare, small scale CNVs. Since many methods have already been published and mutually compared for the analysis of SNP-array data, we decided to use existing methods, instead of creating yet another [24-26,29-31]. The pipeline uses the widely used approach of majority vote based calling, as previously described for the Affymetrix platform [32], to generate a restrictive set of results, with a higher sensitivity and specificity (figure 2). Specificity is increased by discarding CNVs called by only 1 of the 3 methods, usually representing method specific artefacts. An increase in sensitivity over each single method is reached by adding regions that were called by 2 methods, but not by the third. CNV delineation is done with a conservative overlap, retaining only regions seen by more than one method.

Previous studies comparing multiple computational methods for microarray analysis showed that Hidden Markov Model (HMM) based methods perform best in detecting rare and small CNVs. These methods explicitly combine intensity and genotype data, take the 


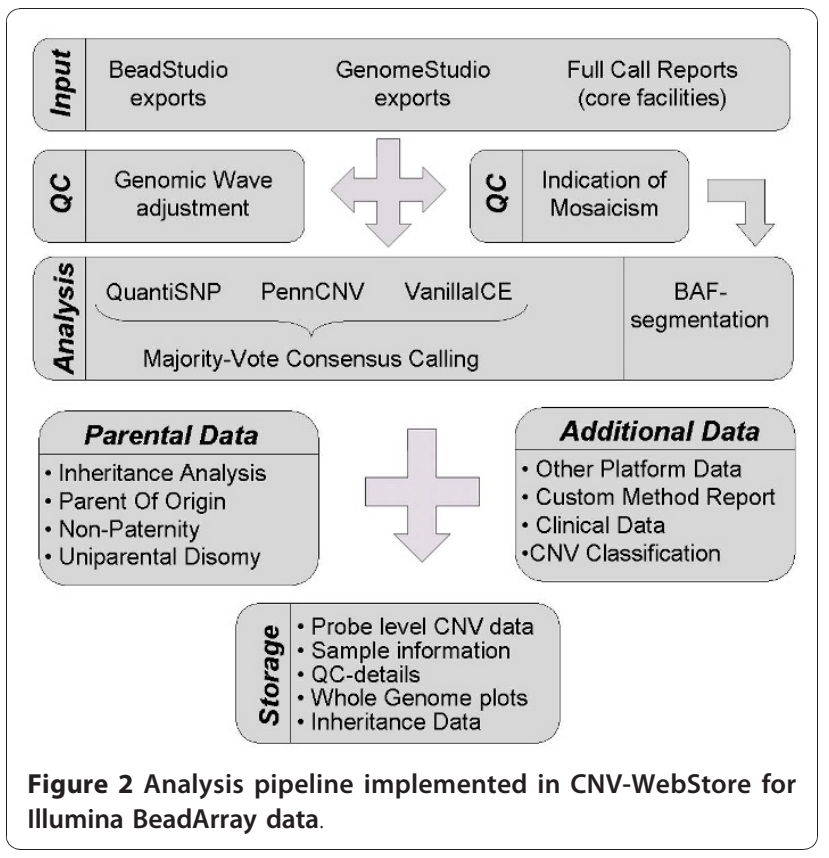

non-uniform probe spacing into account and have an underlying assumption of general diploidy, although they have a significantly larger computational footprint than classic aCGH segmentation methods $[13,26,33]$. In addition, results are sample size independent, in contrast to some recent methods that use a joint calling algorithm over multiple samples [34]. Although joint-calling can be a very powerful tool in GWA projects for the detection of recurrently variable regions, population dependent CNV calls are unacceptable in a clinical context where all results have to be reproducible under any circumstance. We chose QuantiSNP and PennCNV because they are the two most widely accepted and used HMM methods for Illumina data analysis today, and VanillaICE for its claimed high sensitivity to deletions, which are responsible for approximately $70 \%$ of pathogenic events described so far [8].

Besides general copy-number analysis, the data are screened for indications of mosaicism based on quality control parameters, as described below. BAFsegmentation is used with default settings to detect regions of aberrant B-allele frequency, and to estimate the proportion of cells containing the allelic imbalance [27].

Finally, uniparental disomy detection is implemented using the method described by Ting et al. [28]. Based on informative combinations of mendelian inconsistencies, uniparental heterodisomy, isodisomy and non-paternity can be deduced from the provided genotype data.

\section{Performance estimation}

To estimate performance of the proposed majority voting, its stringency and sensitivity were compared against the separate methods using a reference dataset generated by Pinto et al. using an approach similar to ours [32], and a dataset of 192 HapMap samples generated by Illumina on the HumanCNV370-Quad BeadChip, from which 181 samples were also present in the former study. None of the separate methods performed significantly better in picking up the previously published CNV regions from the Illumina data (Table 1). Similar results were obtained using a set of $\mathrm{CNVs}$ seen in a study by McCarroll et al on the Affymetrix 6.0 array [3] (data not shown).

The slight sacrifice in sensitivity towards duplications, caused by incorporation of VanillaICE in the majority vote, can be countered by taking advantage of the observed high power of PennCNV in detecting duplication events. To do so, an option for asymmetric voting, where PennCNV duplication calls do not need to be confirmed by a second method, is available.

When applied to the full panel of 192 high quality HapMap samples, we retrieved a set of 8779 aberrations. This corresponds to a mean of 45 aberrations per sample and to $4 \%$ of the genome present in a variable number of copies over all the samples. This high number of CNVs per sample seems out of range with previous studies. When classified by size or coverage (figure 3 ), it is clear that most of the called CNVs are found in the category of 3-6 consecutive probes. Thus, it appears that the increase in resolution results in an increase in the number of additional small CNVs detected. Indeed, using an artificial resolution of $100 \mathrm{~Kb}$, corresponding to 15 consecutive probes, we detected $8 \mathrm{CNVs}$ per sample, in range with an average of $6 \mathrm{CNVs}$ per sample detected on a $85 \mathrm{~K}$ oligonucleotide platform with a practical resolution of $100 \mathrm{~Kb}[4]$.

\section{Quality Control}

Quality control parameters are stored during analysis and user feedback is given if they exceed preset values. High quality samples are considered to have a call rate of $>99.4 \%$, LogR standard Deviation $<0.2$, B-allele

Table 1 Proportion of CNVs called by Pinto et al. detected by the different methods used

\begin{tabular}{lcccccc}
\hline & $\begin{array}{c}\text { Deletions } \\
(\boldsymbol{n}=\mathbf{3 7 8})\end{array}$ & $\begin{array}{c}\text { Duplications } \\
(\boldsymbol{n}=\mathbf{1 3 0})\end{array}$ & $\begin{array}{c}\text { Overall } \\
(\boldsymbol{n}=\mathbf{5 0 8})\end{array}$ \\
\hline Algorithm & $\%$ & P-Val & $\%$ & P-Val & $\%$ & P-Val \\
\hline 2/3 Vote & 81.5 & & 69.6 & & 74.4 & \\
QuantiSNP & 79.0 & 0.19 & 66.0 & 0.26 & 71.3 & 0.13 \\
PennCNV & 80.0 & 0.3 & 75.2 & 0.16 & 77.2 & 0.15 \\
VanillalCE & 83.0 & 0.31 & 55.1 & 0.0073 & 66.3 & 0.0023 \\
\hline
\end{tabular}

Results are averaged over 181 HapMap samples. P-values are calculated using a one-tailed z-test for difference between proportions, comparing the majority vote against each separate method. 


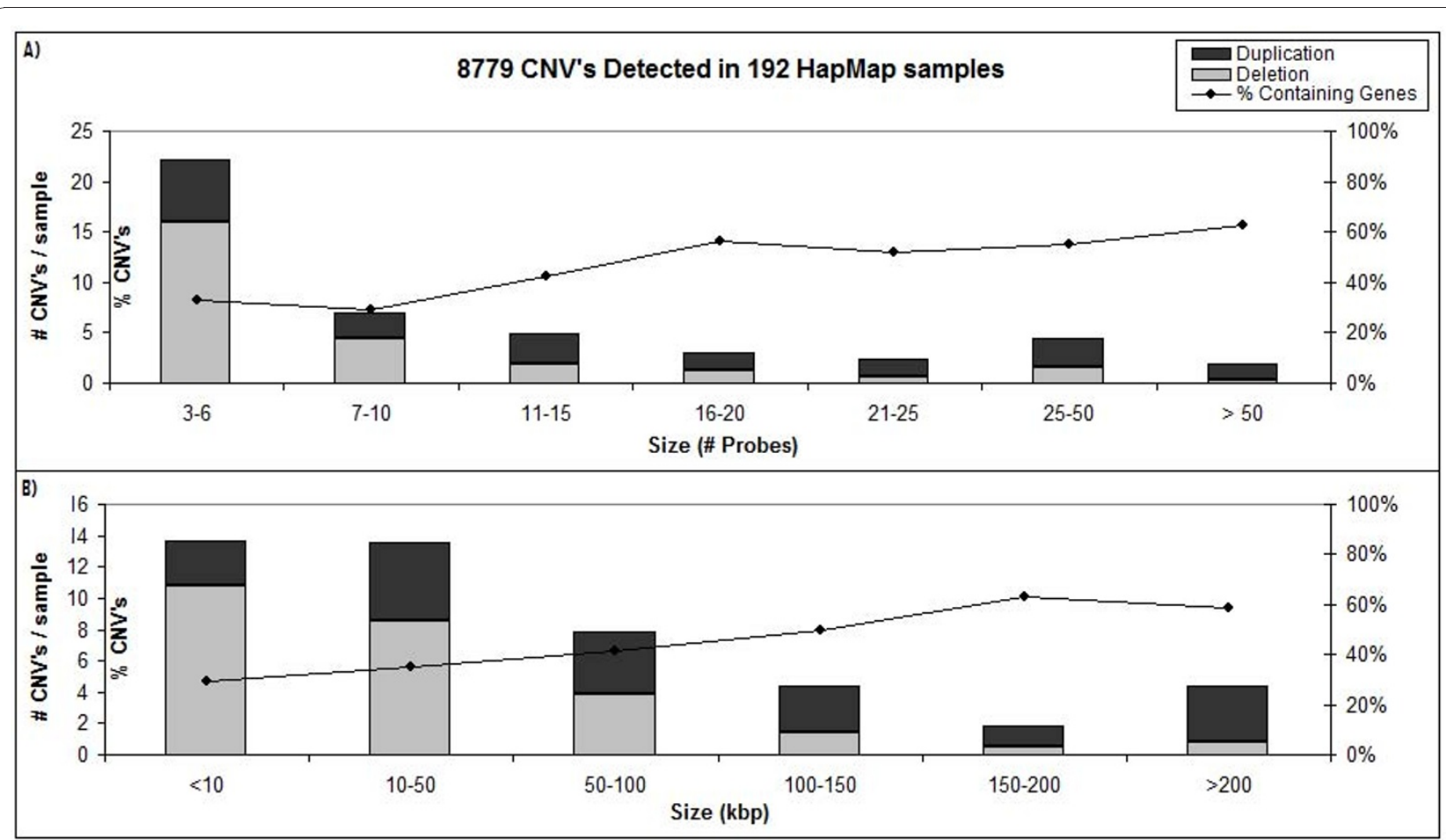

Figure 3 Overview of $\mathbf{8 7 7 9}$ copy number variations detected in $\mathbf{1 9 2}$ HapMap Samples. On the left axis, the number of deletions per sample are shown as light bars, duplications as dark bars. On the right axis the percentage of aberrations in the bin, containing genes is shown. A) Classified by coverage. B) Classified by aberration size.

Standard Deviation $<0.6$ and absolute genomic wave $<0.03$. In case of genomic waving, an event where the intensity data shows typical fluctuation across the chromosomes, normalisation is performed during $\mathrm{CNV}$ analysis [35]. QC-values are taken from PennCNV output.

Chromosome specific B-Allele standard deviation, calculated by QuantiSNP, is screened for significant outliers, as these might be an indication of mosaicism. When mosaicism is suspected, the user is informed of possible mosaicism and BAFsegmentation is started [27].

\section{Other Platform Data}

To import generic CNV reports, custom parsers can be generated for any tab-separated file. Once imported into the database, family relations and raw probe level data can be added. When this raw data is available, inheritance examination and parent of origin analysis can be carried out as described below.

\section{Sample Annotation}

Default annotation of samples consists of quality control data, used platform type, gender and the total number of detected aberrations. Additionally, clinical data can be added. Structured clinical information, conforming to the London neurogenetics nomenclature [36], aids in unambiguous comparison and grouping of samples based on their phenotype.

\section{CNV Annotation}

Clinical interpretation of CNVs requires a comprehensive cross-linking of available annotation data. Besides experimental data such as aberration size, copy number state, inheritance data and genome build, several additional annotations are automatically added (figure 4).

Affected RefSeq genes [37] are listed, with associations to phenotypes extracted from OMIM [38]. Known disease related loci and published case reports are extracted from the DECIPHER and ECARUCA databases [15,39]. Finally, information on flanking segmental duplications is available, as these often flank recurrent microdeletion and -duplication regions [40]. The Toronto Database of Genomic Variants is a major collection of known benign variants detected in control populations [1]. The database comprises a total of almost 40 studies, from which a user can select a subset used for automatic annotation. As a second source of healthy controls, all HapMap samples analysed by Illumina to create the reference cluster files, passing our quality control requirements, were analysed by the described pipeline for all supported chip types and presented to the user as a chip-specific control set [11]. 


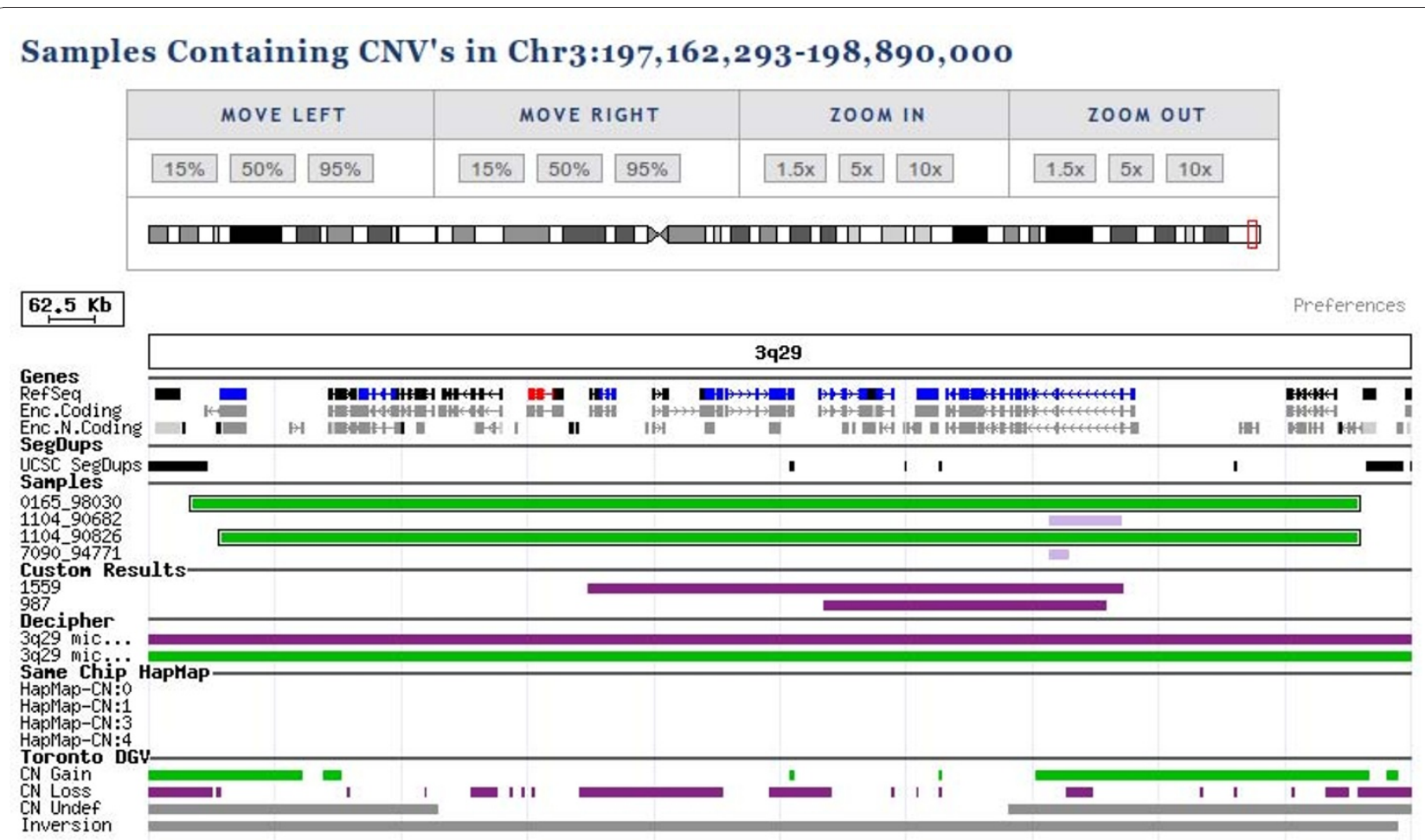

Figure 4 Graphical representation of CNV-annotation by CNV-WebStore. General color codes are green for duplication and purple for deletion events. OMIM information for genes is indicated by blue or red (MORBID) color.

Based on supplied family information, CNVs are further annotated with inheritance information, parent of origin information for de novo events and uniparental iso- or heterodisomy prediction [28].

\section{Data management and protection}

All data are stored in a relational database which is backed up on a daily basis. Access to specific data using CNVWebStore is regulated by user-level permissions. Users are allowed to manage and share their own data with different users or user groups, with varying privileges, ranging from read-only access, over the privilege to only edit clinical or CNV annotations, to the right to further share data with more users. In order to guarantee data integrity, all changes in annotation are kept by a logging system. Sample anonymity is ensured as there is no storage of personal information, such as names or birth dates. Sample identification relies on arbitrary identification codes specified by the user. A typical example would be a numeric identifier linking to the DNA sample that was analysed.

\section{Data Representation}

Centralised storage of all data allows flexible presentation and searching approaches. By default CNVWebStore presents data in a compact graphical browser (see figure 4). CNV regions are denoted by bars using a color code representing copy number and clinical significance. Results from multiple experiments can be combined in a single overview or can be visualised separately. The graphical representation allows intuitive correlation of results with previous results, gene content, known pathogenic regions and benign variants. It further provides access to inheritance information, probe level data for visual examination, quality control results, general sample information and several public resources for extensive investigation of annotation data $[38,41,42]$ (see figure 5). Individual users can select the resources they consider relevant to be included, or add new resources available to all users.

Alternatively, data for a single sample can also be presented in a tabular format. Here, clinical data is bundled with experimental data, CNV annotation, occurrence statistics and reporting tools. Additional annotation tools present from here are a direct PubMed querying tool to intersect the clinical data with gene content, and a CNV prioritization option based on the Endeavour program [19].

\section{Reporting}

The reporting module of CNV-WebStore provides uniform and comprehensive summaries of annotated data. Users have the option to include clinical and experimental 


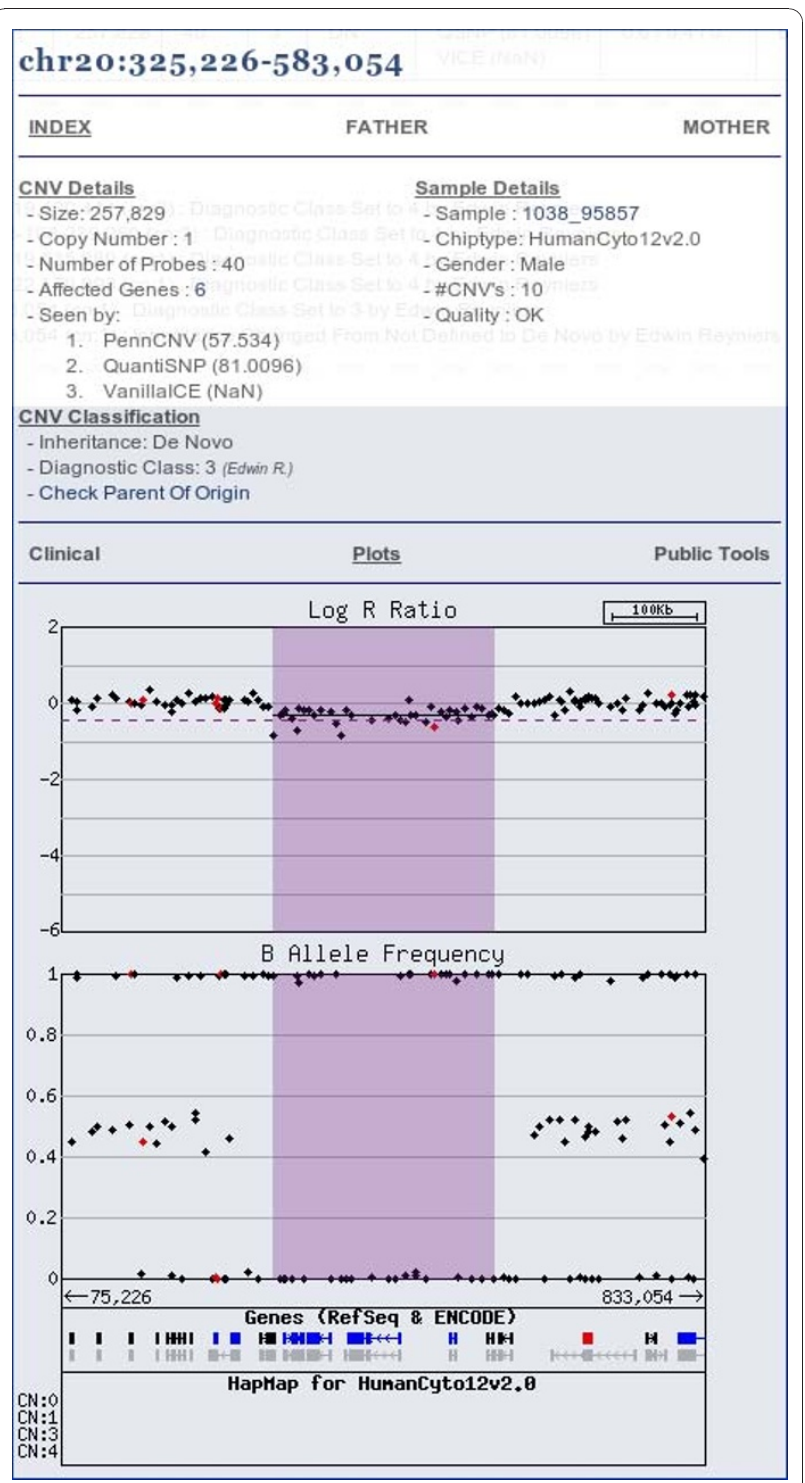

Figure 5 Context Information presentation. A single tooltip allows access to parental data (on top), clinical information and public resources (middle) and visual examination plots (bottom).

details, a schematic karyogram, ISCN representation [43], all or a filtered subset of detected aberrations, gene content and annotation history.

\section{Exporting}

To analyse data using other tools, results can be exported as Tab separated files, BED files compatible with UCSC and Ensembl genome browsers, or XML files compatible with Illumina Genome Viewer.

\section{Discussion}

SNP-based microarray platforms are used more and more in daily diagnostics, providing the potential to analyse a patient's genome at high resolution on both genotype and copy number level. As for many of today's technologies, the amount of produced data is vast and interpretation becomes a cumbersome task.

To overcome this, and to help sieve through the many generated CNV calls, we developed CNV-WebStore, a centralised analysis and interpretation platform, able to go from raw data to reports from a single interface. It implements a majority vote analysis pipeline combining three HMM based methods, each using slightly different underlying models, with a net effect of filtering out model specific artefacts. A similar approach has been successfully applied on Affymetrix 500 K SNP array data, to obtain a stringent $\mathrm{CNV}$ set consisting of the most reliable calls [32].

From our results, two observations were made underscoring that obtaining the correct functional correlation between genotype and phenotype is the major hurdle in routine microarray usage. First, about $30 \%$ of the smallest detectable CNVs on the used platform disrupt 1 or more known coding sequences, often affecting exonic sequence. Second, CNVs affecting only intronic sequence might still contribute to specific phenotypes, as intronic sequences often harbour non-coding transcripts, which are currently emerging as key regulators in many diseases [44-46]. Furthermore, we expect that with the rising probe density of current and future microarray platforms, and with new techniques such as next generation sequencing based CNV calling, the amount of small variants detected will keep increasing, stressing the importance of a straightforward interpretation platform such as CNV-WebStore.

Interpretation tools made available to the user are presented in a unified way, offering maximal information with minimal effort. CNV-WebStore offers both molecular data, automatic CNV annotation and clinical information, in the context of public reference data or previous experimental results. The option to correlate clinical information with extended annotation data from a single view is a feature seen in some public platforms such as DECIPHER, but lacking in other platforms for routine usage [15,21-23]. Furthermore, the option to analyse data from the platform itself, without the need to use a command line interface is a major benefit of our platform over existing, similar interpretation solutions [15,21]. Finally, the capability to infer parent of origin and uniparental disomy from stored genotyping data is a unique feature of CNV-WebStore with clear impact on functional interpretation of imprinting related disorders [47]. A drawback might be that HMM based methods assume integer copy number values, limiting the possible application of the presented analysis pipeline in cancer research [27]. On the other hand, as incorporation of post-analysis results is supported for any type of data, including better suited segmentation 
based methods, the platform can create a substantial added value for interpretation of these data.

By integrating both data analysis, storage and presentation into a single platform, the problem of computational resources might impose a practical limit on usability. To address this, a system monitor is implemented in the analysis pipeline that distributes the system load to those processors that are spare in the system. Cloud computing, as illustrated recently for the Reciprocal Smallest Distance Algorithm, was also considered [48]. As a hosting server with 8 cores and $2 \mathrm{~Gb}$ RAM per core is able to analyse samples with $1 \mathrm{M}$ markers in less than 10 minutes, the actual limit lies in data storage and not in computational power, which is nowadays easily managed, without the large financial overhead of renting cloud-based storage.

\section{Conclusions}

By combining microarray data analysis with centralised storage, a comprehensive start point was created for data interpretation, the major task in current diagnostic usage of microarray data. The ability to compare results against clinical information, previously analysed samples, known variant regions and gene content allows a sensitive selection of CNVs to obtain the most relevant results for further research. Extensive logging enables the tracking of changes to every sample or region, making CNV-WebStore a useful tool in a collaborative setup.

In conclusion, we have developed a web based platform providing an intuitive pipeline to go with minimal effort from raw data to functional interpretation and reporting of results. Because both lab technicians and clinical staff can annotate the data from their own expertise, the most relevant regions will most likely come forward. This makes CNV-WebStore a valuable tool in daily clinical practice, where modern techniques often produce overwhelming amounts of data.

\section{Availability and requirements}

- Project Name: CNV-WebStore

- Project Home Page: http://sourceforge.net/projects/ cnv-webstore

- WebService Address: http://medgen.ua.ac.be/cnv

- Operating Systems: UNIX-like 64bit OS

- Programming Language: Perl/PHP/R/MySQL

- Other Requirements: LAMP server

- License: GNU GPL, free for academic usage

\section{Additional material}

Additional file 1: VanillaICE code and settings. VanillalCE settings were adapted to optimise performance on Illumina BeadChip data. This files contains the resulting R-code that can be used with version 1.4.0.

\section{Acknowledgements}

Funding: Our work is supported by the Belgian National Fund for Scientific Research - Flanders (FWO) and the Marguerite-Marie Delacroix foundation.

\section{Author details}

'Department of Medical Genetics, University Hospital Antwerp, Antwerp, Belgium. ${ }^{2}$ University Hospital Antwerp, Antwerp, Belgium.

\section{Authors' contributions}

GV developed the software platform described and wrote the manuscript. ER and LR performed validation studies and contributed to the conceptual design of the platform. WW contributed to the conceptual design of the platform. RFK supervised the project and the final manuscript. All authors read and approved the final manuscript.

Received: 18 August 2010 Accepted: 5 January 2011

Published: 5 January 2011

\section{References}

1. lafrate AJ, Feuk L, Rivera MN, Listewnik ML, Donahoe PK, Qi Y, Scherer SW, Lee C: Detection of large-scale variation in the human genome. Nat Genet 2004, 36(9):949-951.

2. Redon $R$, Ishikawa S, Fitch KR, Feuk L, Perry GH, Andrews TD, Fiegler $H$, Shapero MH, Carson AR, Chen W, et al: Global variation in copy number in the human genome. Nature 2006, 444(7118):444-454.

3. McCarroll SA, Kuruvilla FG, Korn JM, Cawley S, Nemesh J, Wysoker A, Shapero MH, de Bakker PI, Maller JB, Kirby A, et al: Integrated detection and population-genetic analysis of SNPs and copy number variation. Nat Genet 2008, 40(10):1166-1174.

4. Walsh T, McClellan JM, McCarthy SE, Addington AM, Pierce SB, Cooper GM, Nord AS, Kusenda M, Malhotra D, Bhandari A, et al: Rare structural variants disrupt multiple genes in neurodevelopmental pathways in schizophrenia. Science (New York, NY 2008, 320(5875):539-543.

5. Yoo SM, Choi JH, Lee SY, Yoo NC: Applications of DNA microarray in disease diagnostics. Journal of microbiology and biotechnology 2009, 19(7):635-742.

6. Marshall CR, Noor A, Vincent JB, Lionel AC, Feuk L, Skaug J, Shago M, Moessner R, Pinto D, Ren $Y$, et al: Structural variation of chromosomes in autism spectrum disorder. Am J Hum Genet 2008, 82(2):477-488.

7. Lanktree M, Hegele RA: Copy number variation in metabolic phenotypes. Cytogenet Genome Res 2008, 123(1-4):169-175.

8. Koolen DA, Pfundt R, de Leeuw N, Hehir-Kwa JY, Nillesen WM, Neefs 1 , Scheltinga I, Sistermans E, Smeets D, Brunner HG, et al: Genomic microarrays in mental retardation: a practical workflow for diagnostic applications. Hum Mutat 2009, 30(3):283-292.

9. McMullan DJ, Bonin M, Hehir-Kwa JY, de Vries BBA, Dufke A, Rattenberry E, Steehouwer M, Moruz L, Pfundt R, de Leeuw N, et al: Molecular karyotyping of patients with unexplained mental retardation by SNP arrays: a multicenter study. Hum Mutat 2009, 30(7):1082-1092.

10. Snijders AM, Nowak N, Segraves R, Blackwood S, Brown N, Conroy J, Hamilton G, Hindle AK, Huey B, Kimura K, et al: Assembly of microarrays for genome-wide measurement of DNA copy number. Nat Genet 2001, 29(3):263-264.

11. Peiffer DA, Le JM, Steemers FJ, Chang W, Jenniges T, Garcia F, Haden K, Li J, Shaw CA, Belmont J, et al: High-resolution genomic profiling of chromosomal aberrations using Infinium whole-genome genotyping. Genome Res 2006, 16(9):1136-1148.

12. Stankiewicz P, Beaudet AL: Use of array CGH in the evaluation of dysmorphology, malformations, developmental delay, and idiopathic mental retardation. Curr Opin Genet Dev 2007, 17(3):182-192.

13. Yu T, Ye H, Sun W, Li KC, Chen Z, Jacobs S, Bailey DK, Wong DT, Zhou X: A forward-backward fragment assembling algorithm for the identification of genomic amplification and deletion breakpoints using high-density single nucleotide polymorphism (SNP) array. BMC bioinformatics 2007, 8:145.

14. Hester SD, Reid L, Nowak N, Jones WD, Parker JS, Knudtson K, Ward W, Tiesman J, Denslow ND: Comparison of comparative genomic hybridization technologies across microarray platforms. J Biomol Tech 2009, 20(2):135-151.

15. Firth HV, Richards SM, Bevan AP, Clayton S, Corpas M, Rajan D, Van Vooren S, Moreau Y, Pettett RM, Carter NP: DECIPHER: Database of Chromosomal Imbalance and Phenotype in Humans Using Ensembl Resources. Am J Hum Genet 2009, 84(4):524-533. 
16. Aerts $S$, Lambrechts $D$, Maity $S$, Van Loo P, Coessens B, De Smet F, Tranchevent L-C, De Moor B, Marynen P, Hassan B, et al: Gene prioritization through genomic data fusion. Nat Biotechnol 2006, 24(5):537-544

17. Qiao Y, Harvard C, Tyson C, Liu X, Fawcett C, Pavlidis P, Holden JJ, Lewis ME, Rajcan-Separovic E: Outcome of array CGH analysis for 255 subjects with intellectual disability and search for candidate genes using bioinformatics. Hum Genet 128(2):179-194.

18. Lai WR, Johnson MD, Kucherlapati R, Park PJ: Comparative analysis of algorithms for identifying amplifications and deletions in array CGH data. Bioinformatics (Oxford, England) 2005, 21(19):3763-3770.

19. Tranchevent LC, Barriot R, Yu S, Van Vooren S, Van Loo P, Coessens B, De Moor B, Aerts S, Moreau Y: ENDEAVOUR update: a web resource for gene prioritization in multiple species. Nucleic Acids Res 2008, , 36 Web Server: W377-384.

20. Hehir-Kwa JY, Wieskamp N, Webber C, Pfundt R, Brunner HG, Gilissen C, de Vries $B B$, Ponting $C P$, Veltman JA: Accurate distinction of pathogenic from benign CNVs in mental retardation. PLoS computational biology 2010, 6(4): e1000752.

21. Gai X, Perin JC, Murphy K, O'Hara R, D'Arcy M, Wenocur A, Xie HM, Rappaport EF, Shaikh TH, White PS: CNV Workshop: an integrated platform for high-throughput copy number variation discovery and clinical diagnostics. BMC bioinformatics 2010, 11:74.

22. Kim SY, Nam SW, Lee SH, Park WS, Yoo NJ, Lee JY, Chung YJ: ArrayCyGHt: a web application for analysis and visualization of array-CGH data. Bioinformatics (Oxford, England) 2005, 21(10):2554-2555.

23. Menten B, Pattyn F, De Preter K, Robbrecht P, Michels E, Buysse $K$ Mortier G, De Paepe A, van Vooren S, Vermeesch J, et al: arrayCGHbase: an analysis platform for comparative genomic hybridization microarrays. BMC bioinformatics 2005, 6:124

24. Colella S, Yau C, Taylor JM, Mirza G, Butler H, Clouston P, Bassett AS, Seller A, Holmes CC, Ragoussis J: QuantiSNP: an Objective Bayes Hidden-Markov Model to detect and accurately map copy number variation using SNP genotyping data. Nucleic Acids Res 2007, 35(6):2013-2025

25. Wang K, Li M, Hadley D, Liu R, Glessner J, Grant SF, Hakonarson H, Bucan M: PennCNV: an integrated hidden Markov model designed for highresolution copy number variation detection in whole-genome SNP genotyping data. Genome Res 2007, 17(11):1665-1674.

26. Scharpf RB, Parmigiani G, Pevsner J, Ruczinski I: Hidden Markov models for the assessment of chromosomal alterations using high-throughput SNP arrays. The annals of applied statistics 2008, 2(2):687-713.

27. Staaf J, Lindgren D, Vallon-Christersson J, Isaksson A, Goransson $H_{\text {, }}$ Juliusson G, Rosenquist R, Hoglund M, Borg A, Ringner M: Segmentationbased detection of allelic imbalance and loss-of-heterozygosity in cancer cells using whole genome SNP arrays. Genome Biol 2008, 9(9):R136.

28. Ting JC, Roberson ED, Miller ND, Lysholm-Bernacchi A, Stephan DA, Capone GT, Ruczinski I, Thomas GH, Pevsner J: Visualization of uniparental inheritance, Mendelian inconsistencies, deletions, and parent of origin effects in single nucleotide polymorphism trio data with SNPtrio. Hum Mutat 2007, 28(12):1225-1235.

29. Sun W, Wright FA, Tang Z, Nordgard SH, Loo PV, Yu T, Kristensen VN, Perou CM: Integrated study of copy number states and genotype calls using high-density SNP arrays. Nucleic Acids Res 2009.

30. Yavas G, Koyuturk M, Ozsoyoglu M, Gould MP, LaFramboise T: An optimization framework for unsupervised identification of rare copy number variation from SNP array data. Genome Biol 2009, 10(10):R119.

31. Lin $M$, Wei LJ, Sellers WR, Lieberfarb $M$, Wong WH, Li C: dChipSNP: significance curve and clustering of SNP-array-based loss-ofheterozygosity data. Bioinformatics (Oxford, England) 2004, 20(8):1233-1240.

32. Pinto D, Marshall C, Feuk L, Scherer SW: Copy-number variation in control population cohorts. Hum Mol Genet 2007, 16(Spec No 2):R168-173.

33. Yau C, Holmes CC: CNV discovery using SNP genotyping arrays. Cytogenet Genome Res 2008, 123(1-4):307-312

34. Alonso A, Julia A, Tortosa R, Canaleta C, Canete JD, Ballina J, Balsa A, Tornero J, Marsal S: CNstream: A method for the identification and genotyping of copy number polymorphisms using Illumina microarrays. BMC bioinformatics 2010, 11(1):264.

35. Diskin SJ, Li M, Hou C, Yang S, Glessner J, Hakonarson H, Bucan M, Maris JM, Wang K: Adjustment of genomic waves in signal intensities from whole-genome SNP genotyping platforms. Nucleic Acids Res 2008, 36(19):e126.

36. Fryns JP, de Ravel TJ: London Dysmorphology Database, London Neurogenetics Database and Dysmorphology Photo Library on CD-ROM [Version 3] 2001R. M. Winter, M. Baraitser, Oxford University Press, ISBN 019851-780, pound sterling 1595. Hum Genet 2002, 111(1):113.

37. Pruitt KD, Tatusova T, Klimke W, Maglott DR: NCBI Reference Sequences: current status, policy and new initiatives. Nucleic Acids Res 2009, , 37 Database: D32-36

38. Hamosh A, Scott AF, Amberger JS, Bocchini CA, McKusick VA: Online Mendelian Inheritance in Man (OMIM), a knowledgebase of human genes and genetic disorders. Nucleic Acids Res 2005, , 33 Database: D514-517.

39. Feenstra I, Fang J, Koolen DA, Siezen A, Evans C, Winter RM, Lees MM, Riegel M, de Vries BB, Van Ravenswaaij CM, et al: European Cytogeneticists Association Register of Unbalanced Chromosome Aberrations (ECARUCA); an online database for rare chromosome abnormalities. Eur $J$ Med Genet 2006, 49(4):279-291.

40. Lupski JR, Stankiewicz P: Genomic disorders: molecular mechanisms for rearrangements and conveyed phenotypes. PLoS Genet 2005, 1(6):e49.

41. Rebholz-Schuhmann D, Kirsch H, Arregui M, Gaudan S, Riethoven M, Stoehr P: EBIMed-text crunching to gather facts for proteins from Medline. Bioinformatics (Oxford, England) 2007, 23(2):e237-244.

42. Hoffmann R: A wiki for the life sciences where authorship matters. Nat Genet 2008, 40(9):1047-1051.

43. Shaffer LGSM, Campbell LJ: ISCN 2009: An International System for Human Cytogenetic Nomenclature. 2009, 126.

44. Hinske LC, Galante PA, Kuo WP, Ohno-Machado L: A potential role for intragenic miRNAs on their hosts' interactome. BMC Genomics 2010, 11:533.

45. White NM, Yousef GM: MicroRNAs: exploring a new dimension in the pathogenesis of kidney cancer. BMC Med 2010, 8(1):65.

46. Gatto S, Ragione FD, Cimmino A, Strazzullo M, Fabbri M, Mutarelli M, Ferraro L, Weisz A, D'Esposito M, Matarazzo MR: Epigenetic alteration of microRNAs in DNMT3B-mutated patients of ICF syndrome. Epigenetics 2010, 5(5)

47. Yamazawa K, Ogata T, Ferguson-Smith AC: Uniparental disomy and human disease: an overview. Am J Med Genet C Semin Med Genet 2010, 154C(3):329-334

48. Wall DP, Kudtarkar P, Fusaro VA, Pivovarov R, Patil P, Tonellato PJ: Cloud computing for comparative genomics. BMC bioinformatics 2010, 11:259.

\section{doi:10.1186/1471-2105-12-4}

Cite this article as: Vandeweyer et al:: CNV-WebStore: Online CNV Analysis, Storage and Interpretation. BMC Bioinformatics 2011 12:4

\section{Submit your next manuscript to BioMed Central and take full advantage of:}

- Convenient online submission

- Thorough peer review

- No space constraints or color figure charges

- Immediate publication on acceptance

- Inclusion in PubMed, CAS, Scopus and Google Scholar

- Research which is freely available for redistribution

Submit your manuscript at www.biomedcentral.com/submit
C Biomed Central 